\title{
PRODUCCIÓN ACADÉMICA EN ESTUDIOS DE LA MUJER (1996-2000)
}

\section{Mirta González Suárez*}

\author{
RESUMEN
}

El artículo presenta un seguimiento de la producción en estudios de la mujer y género, de acuerdo con el número de proyectos de investigación, tesis, libros y artículos científicos de las cuatro universidades públicas, durante los años 1996-2000, que llegan a un total de 381 documentos. Los temas más destacados son educación, economía y trabajo, política, violencia, familia, literatura y feminismo. Aún cuando los anteriores son una respuesta adecuada a las demandas de las organizaciones todavía hay déficit de estudios sobre tópicos tan relevantes como pobreza, sexualidad $y$ derechos reproductivos.

PALABRAS CLAVES: GÉNERO * MUJERES * ESTUDIOS DE LA MUJER * CIENCIA * ACADEMIA

\section{ABSTRACT}

This article presents data based on the number of research projects, dissertations, books and articles related to Women's Studies and gender issues written at the four state universities, during 1996-2000. This follow up finds a total of 381 documents. The highlights topics are education, health, economy and work, political participation, violence, family, literature and feminism. Even though these include most of the research needs requested by NGO'S there is a lack of studies on major social issues such as poverty, sexuality and reproductive rights.

KEY WORDS: GENDER $*$ WOMEN $*$ WOMEN'S STUDIES $*$ SCIENCE $*$ ACADEMY

\section{INTRODUCCIÓN}

Los Estudios de la Mujer se han desarrollado sistemáticamente en Costa Rica y actualmente cada universidad pública tiene instancias específicas promotoras de la equidad de género, a saber: Centro de Investigación de Estudios de la Mujer (Universidad de Costa Rica, UCR); Instituto de Estudios de la Mujer (Universidad Nacional, UNA) y Programas de Equidad de Género (Instituto Tecnológico de Costa Rica, ITCR y Universidad Estatal a Distancia, UNED). Se cuenta además con el Programa de Posgrado Regional en Estudios de la Mujer con dos

CIEM y Escuela de Psicología, Universidad de Costa Rica. mirtag@amnet.co.cr 
maestrías conjuntas UCR-UNA: la Maestría en Estudios de la Mujer (académica) y la Maestría en Violencia Intrafamiliar y de género (profesional).

Desde la década de los setenta, especialmente después del Año Internacional de la Mujer (1975), se realiza una gran producción en Estudios de la Mujer, a la que periódicamente se le ha dado seguimiento. Como antecedentes básicos en este proceso se encuentran:

$\diamond$ Estudios de la Mujer: Conocimiento y Cambio (Mirta González Suárez,1988) donde se señalan diversas etapas de desarrollo en los Estudios de la Mujer, así como la importancia de monitorear los aportes realizados. El estudio considera hasta 1987, con un total de 454 publicaciones. El aumento significativo se aprecia al considerar que hasta 1960 se detectan sólo 15 publicaciones, mientras que en los ochenta (hasta 1987) la suma asciende a 249. El tema más estudiado fue trabajo, con 137 indicaciones, seguido por temas generales, política y organización, familia y educación.

$\diamond$ Necesidades de la investigación en Estudios de la Mujer en Centroamérica (Mirta González Suárez, 1997) recaba información sobre las demandas de investigación desde la perspectiva de las organizaciones sociales. Para Costa Rica los temas más solicitados (en orden de importancia) fueron: trabajo, violencia, poder, economía, salud, educación, familia e identidad de género.

$\diamond$ El estado de la investigación en Estudios de la Mujer en la Universidad de Costa Rica (Laura Guzmán Stein, 1999) explora los temas inscritos en la Vicerrectoría de Investigación entre 1990-1999 y concluye que los más investigados en la década son: género y salud, identidades de género (masculinidad y feminidad, construcción de identidades en mujeres mayores $y$ adolescentes), trabajo $y$ producción (mujeres rurales y urbanas, adultas y adolescentes), organización, participación política, liderazgo y poder, socialización, ideología y representaciones sociales, género en educación, violencia contra las mujeres, mujeres en la historia, indicadores socioeconómicos, estado de la investigación en Estudios de la Mujer, comunicación, género y mujeres en la universidad.
Las investigaciones indicadas señalan oportunamente la pertinencia de establecer un seguimiento permanente de los cambios ocurridos, por lo que en esta etapa se presentan los datos del último quinquenio del Siglo Xx.

Este artículo corresponde a resultados parciales del proyecto 824-A1-035 del CIEM, titulado La Investigación en Estudios de la Mujer en Costa Rica: avances e inequidades a lo interno de las universidades públicas y capacidad de respuesta entre las demandas de la sociedad civil y las políticas públicas, el que, como su título lo indica, establece relaciones entre la producción académica, las demandas de las organizaciones y las respuestas desde el Estado. La pregunta central se refiere al primer aspecto: la producción universitaria relacionada con Estudios de la Mujer, incluyéndose aquellos estudios de todas las áreas que especifiquen un interés por la situación de las mujeres o señalen la perspectiva de género - por supuesto, incluyendo el tema de la masculinidad- como parte integrante de su quehacer. Cabe señalar que tal opción plantea limitaciones, e incluso en ocasiones podría inducir a errores, ya que, por ejemplo, una publicación centrada en la vida de las mujeres no necesariamente plantea una posición adecuada con respecto a sus derechos o es un avance en la desestructuración del patriarcado. El procedimiento asumido, sin embargo, permite comparar los resultados con investigaciones anteriores a la vez que provee datos globales que responde a los intereses planteados.

\section{PROCEDIMIENTO}

\section{BASE DE DATOS}

Se organiza una base de datos (Access) que incluye los siguientes componentes de las universidades estatales:

\section{INVESTIGACIONES}

$\diamond$ Proyectos de investigación de Estudios de la Mujer inscritos en la instancia respectiva, vicerrectorías de investigación o similar.

$\diamond$ Tesis sobre Estudios de la Mujer. 


\section{PUBLICACIONES}

$\diamond$ Libros publicados sobre Estudios de la Mujer

$\diamond$ Artículos en revistas de cada universidad, sobre Estudios de la Mujer

En todos los casos el estudio se centra en el quinquenio de fin de siglo(1996-2000).

Las fuentes se recogen de instancias oficiales de las universidades, debiendo señalar que en algunas oportunidades no se ofrecieron el total de los datos requeridos y en otras no existía una categorización compatible, por lo que se requirió la revisión manual del material.

\section{CLASIFICACIÓN DE DATOS}

La clasificación se dividió por temas y considerando las áreas señaladas por las conferencias de acción mundial (Beijing y Beijing +5 ), que proveen de un marco que permite verificar la interrelación entre las demandas de cambio y los estudios que se convierten en indicadores de los mismos.

Los datos se clasificaron en dos grandes grupos: investigaciones y publicaciones, entendiendo que en ambos casos se trata de producción y divulgación de conocimientos en Estudios de la Mujer.

Cada grupo fue a su vez subdividido en dos aspectos:

Temas: Los temas obtenidos en la investigación titulada "Necesidades de Investigación en Estudios de la Mujer en Centroamérica" sirvieron de base para la clasificación. Dichos tópicos fueron extraídos de la consulta a diversas organizaciones gubernamentales $y$ no gubernamentales sobre sus requerimientos de investigaciones sobre Estudios de la Mujer.

ÁREAS DE LA PLATAFORMA MUNDIAL DE ACCIÓN DE BEIJING

La Plataforma indica 12 áreas principales para el avance de las mujeres, a saber:

1) Pobreza

2) Educación y capacitación de las mujeres
3) La salud de las mujeres

4) La violencia contra las mujeres

5) Las mujeres $y$ los conflictos armados

6) Las mujeres y la economía

7) Las mujeres en el ejercicio del poder y la adopción de decisiones

8) Mecanismos institucionales para el adelanto de las mujeres

9) Los derechos humanos de las mujeres

10) Las mujeres y los medios de difusión

11) Las mujeres y el medio ambiente

12)Las niñas $y$ adolescentes.

De tratarse de productos que incluyen diversos aspectos se opta por escoger solamente uno, el que se considere más destacado, con el objetivo de no crear la ilusión de numerosas investigaciones debido a la complejidad o perspectiva holística.

\section{RESULTADOS}

A. Tal como se ha indicado los resultados obtenidos parten de la elaboración de una base de datos con información sobre la producción en las cuatro universidades estatales, en el quinquenio de fin de siglo, referente a investigaciones $y$ publicaciones sobre Estudios de la Mujer (Revistas y Libros).

B. Las investigaciones $y$ publicaciones fueron clasificadas de acuerdo con temas centrales y la Plataforma de Acción de Beijing.

A continuación un reporte general de los datos obtenidos:

\section{CUADRO 1}

ESTUDIOS DE LA MUJER (1996-2000) PRODUCCIÓN ACADÉMICA

\begin{tabular}{lrrrrr}
\hline & TOTAL & $\begin{array}{c}\text { AUTORÍA: } \\
\text { MUJERES }\end{array}$ & $\%$ & $\begin{array}{c}\text { AUTORÍA: } \\
\text { HOMBRES }\end{array}$ & $\%$ \\
\hline Investigaciones & 92 & 78 & 85 & 14 & 15 \\
Tesis & 94 & 80 & 85 & 14 & 15 \\
Libros & 51 & 31 & 60 & 20 & 40 \\
Revistas & 144 & 121 & 85 & 23 & 15 \\
\hline TOTAL & 381 & 310 & 80 & 71 & 20 \\
\hline
\end{tabular}




\section{INVESTIGACIONES SOBRE ESTUDIOS DE LA MUJER}

En esta clasificación se consignan las investigaciones oficialmente reportadas por las universidades públicas sobre Estudios de la Mujer, sean estas realizadas por mujeres $u$ hombres. Se especifica que se consideraron sólo las registradas en las instancias pertinentes, ya que es usual en el medio académico realizar investigaciones por interés propio, sin que conste en los medios oficiales a tal efecto, por lo que son sumamente difíciles de constatar hasta su publicación. La determinación de autoría se designa según el o la investigadora principal.

La producción universitaria indica los temas más estudiados, así como las carencias, por lo que es relevante cuantificar las tendencias antedichas.

\section{INVESTIGACIONES}

El total de investigaciones realizadas durante cinco años y reconocidas oficialmente por las universidades públicas ascienden a 92 (ver cuadro 1). La división por sexo de las investigaciones indica que el 85\% de las clasificadas en Estudios de la Mujer son realizadas por mujeres.

\section{CUADRO 2}

INVESTIGACIONES: TEMAS MÁS INVESTIGADOS

\begin{tabular}{lc}
\hline TEMA N:92 & CANTIDAD \\
\hline Educación & 24 \\
Marco teórico/feminismo & 13 \\
Salud & 13 \\
\hline
\end{tabular}

CUADRO 3

INVESTIGACIONES: TEMAS MÁS INVESTIGADOS SEGÚN LA PLATAFORMA DE ACCIÓN DE BEIJING

\begin{tabular}{lc}
\hline TEMA N:81 & CANTIDAD \\
\hline Educación & 25 \\
Salud & 15 \\
La niña/adolescente & 9 \\
\hline
\end{tabular}

Las investigaciones sobre educación, marco teórico y salud cubren el $43 \%$ del total, $y$ de estos un $88 \%$ corresponde a la Plataforma de Acción de Beijing. En esta última los temas relacionados con educación, salud y la niña/ adolescente cubren el $60 \%$ de los estudios.

La diferencia entre las universidades consiste en la preponderancia del tema de educación, especialmente en el ITCR, mientras que en la UCR destacan las investigaciones en salud y en la UNA sobre marco teórico-feminismo.

TESIS

Las tesis de grado son investigaciones realizadas por estudiantes, con la asesoría de profesionales con amplia experiencia, por lo que constituyen aportes importantes para el desarrollo del conocimiento. En total se encontraron 94 (ver cuadro 1).

En las tesis, cuya autoría por sexo se obtiene del primer nombre indicado, se mantiene la tendencia anterior de contar con un $85 \%$ de mujeres trabajando en temas relacionados con Estudios de la Mujer. Igualmente se repite la prevalencia de la UCR con aproximadamente la mitad de las tesis. La distribución es la siguiente:

\section{CUADRO 4}

TESIS: TEMAS MÁS INVESTIGADOS

\begin{tabular}{lc}
\hline TEMA N:94 & CANTIDAD \\
\hline Derechos-trabajo & 16 \\
Poder $y$ política & 8 \\
Violencia & 7 \\
Familia & 7 \\
\hline
\end{tabular}

CUADRO 5

TESIS: TEMAS MÁS INVESTIGADOS SEGÚN LA PLATAFORMA DE ACCIÓN DE BEIJING

\begin{tabular}{lc}
\hline TEMA N:75 & CANTIDAD \\
\hline Violencia & 14 \\
La mujer y la economía & 11 \\
La mujer en el ejercicio del poder & 11 \\
\hline
\end{tabular}


El 80\% de los temas generales pueden ser ubicados también como parte de la Plataforma de Acción. Los cuatro temas mayoritarios ocupan el 40\% del total general. Con respecto a la Plataforma de Acción de Beijing se presentan tres temas que corresponden al 48\% del total.

Comparando las tesis con las investigaciones, se observan diferencias como la preponderancia de aspectos económicos y del poder en los trabajos finales de graduación, mientras que en las segundas se otorga mayor relevancia a aspectos educativos.

Por su relación directa con los objetivos planteados, se presenta a continuación un apartado especial dedicado a la producción de la Maestría Regional en Estudios de la Mujer, UCRUNA, denominada por sus siglas MAEM.

\section{TESIS DE MAESTRÍA EN ESTUDIOS \\ DE LA MUJER: UCR-UNA (MAEM)}

(Presentación en orden cronológico, con asignación de clasificación por tema y según la Plataforma de acción).

1. Salas Torres, Maricel. "Los significados psicosociales del acoso sexual a las mujeres en el ambiente de trabajo: el caso del ICE". (1996) (Desarrollo económico/trabajo-violencia contra las mujeres).

2. Villarreal Montoya, Cecilia. "La menopausia: un proceso de crecimiento". (1996) (Saludla mujer y la salud).

3. Brenes Solórzano, Irene. "Relaciones de poder entre los géneros: cambios derivados de la organización y la participación en proyectos productivos. El caso de las mujeres del Jardín de Niños de Pérez Zeledón" (1997)(Desarrollo económico/trabajo ruralla mujer y la economía).

4. Camacho Rojas, Fresia. "Género y comunicación: El caso del programa de radio En la Misma Onda". (1997) (Medios de comunicación-medios de difusión).

5. Zuñiga Bolaños, Rosario. "La extensión forestal, el género y las mujeres desde las representaciones de los/las extensionistas: Estudio de caso en León, Nicaragua". (1998) (Desarrollo económico/trabajo-La mujer y el medio ambiente).
6. Solano Arias, Marta. "Las sufragistas de la Liga Feminista”. (1998) (Política Pública/ Toma de decisión-La mujer en el ejercicio del poder y la toma de decisiones).

7. Jiménez Chaves, Blanca Luz. "Experiencias laborales de las mujeres que trabajan en la cárcel”. (1998) (Desarrollo económico/trabajo- los derechos humanos de las mujeres).

8. Cabrera Víquez, María Alexandra. "Las representaciones sociales femeninas y sus contradicciones en la vida cotidiana”. (1999) (Identidad de género-los derechos humanos de la mujer).

9. Marenco Marrochi, Leda. "El trabajo industrial domiciliario en Costa Rica: una relación estrecha entre la clase social y la construcción de género". (2000) (Desarrollo económico/trabajo-la mujer y la economía).

10.Flansburg, Sundra. "Los derechos reproductivos y sexuales. Concepciones de algunas mujeres costarricenses". (2000) (Derechos reproductivos-la mujer y la salud).

Tal como se puede apreciar en las tesis de la MAEM el tema de trabajo ocupa la mitad de las investigaciones. Desde la plataforma de acción se cubren más de la mitad de los temas señalados como problemáticos $y$, es evidente la variedad de temas en ambas divisiones.

\section{PUBLICACIONES SOBRE ESTUDIOS DE LA MUJER}

Las publicaciones sobre Estudios de la Mujer se obtuvieron de fuentes oficiales de las universidades públicas, especialmente las editoriales y las bibliotecas respectivas.

Se dividieron en libros y revistas, tomando como autoría, para efectos de clasificación por sexo, el nombre que aparece primero, ya que se desea cuantificar la cantidad de publicaciones y no el número de participantes.

\section{LIBROS}

En total se encontraron 51 libros (ver cuadro 1). Mientras que tanto en investigaciones, tesis y revistas sobre Estudios de la Mujer la autoría femenina asciende al 85\%, 
en los libros dicha cantidad disminuye a un $60 \%$. Además hay que considerar que un factor central de escogencia era el título, lo que en el caso de las novelas, constituye una debilidad de procedimiento, ya que la usual utilización de nombres de mujeres no necesariamente indica la tendencia a estudiar las diferencias de género o a cuestionar estereotipos.

Los resultados reflejan una amplia preponderancia de la producción literaria y artística, con un total de 35 volúmenes sobre arte $y$ cultura, lo que corresponde a un $68 \%$ del total.

Los libros que abarcan temas relacionados con la Plataforma corresponden únicamente al 24\%,y entre estos el 58\% corresponden a Educación.

Del total de libros publicados el $72 \%$ corresponde a la Editorial de la Universidad de Costa Rica.

Tenemos así que las publicaciones de libros, en el ámbito de interés, se centran en literatura y educación, lo que denota una concentración y omisión de temas indicados como necesarios desde una perspectiva del avance en los derechos.

Con respecto a artículos en revistas académicas, los resultados son los siguientes:

\section{REVISTAS}

En total se encontraron 144 artículos en revistas (ver cuadro 1).

La proporción por sexo regresa al 85\% realizado por mujeres, tal como se obtuvo en investigaciones y tesis. La producción de la UCR, si bien disminuye unos puntos (47\%), se mantiene en alrededor del doble del resto en su conjunto.

Los temas indicados constituyen el $49 \%$ del total. Comparando los resultados de las

CUADRO 6

PUBLICACIONES EN REVISTAS: TEMAS MÁS INVESTIGADOS

\begin{tabular}{lc}
\hline TEMAS N:144 & CANTIDAD \\
\hline Educación & 27 \\
Cultura, arte & 25 \\
Marco teórico-feminismo & 18 \\
\hline
\end{tabular}

publicaciones, la cultura y arte (tal vez por contar con revistas específicas) cuentan con un alto porcentaje, así como los temas de educación. El desarrollo teórico, sobre todo desde el feminismo, refleja el interés por cuestionar la ciencia androcéntrica y se puede considerar como un paso importante.

De todos los temas un 60\% corresponde a rubros de la Plataforma, lo que implica un aumento con respecto a los libros, pero se mantiene una diferencia de alrededor del $20 \%$ con relación a las investigaciones.

Los tres temas indicados constituyen el $49 \%$ del total, persistiendo la relevancia de la educación.

\section{CUADRO 7}

PUBLICACIONES EN REVISTAS: TEMAS MÁS INVESTIGADOS SEGÚN LA PLATAFORMA DE ACCIÓN DE BEIJING

\begin{tabular}{lc}
\hline TEMAS: N: 87 & CANTIDAD \\
\hline Educación & 26 \\
La mujer en el ejercicio del poder & 16 \\
La violencia & 11 \\
\hline
\end{tabular}

\section{DISCUSIÓN}

\section{INVESTIGACIONES}

Las investigaciones ascienden a 92, de las cuales el 55\% corresponde a la Universidad de Costa Rica y del total el 85\% son dirigidas por mujeres. El tema que más sobresale es Educación, seguido por marco teórico/feminismo y salud. El 53\% de las investigaciones se ubican en algún rubro de la Plataforma Mundial de Acción, sobresaliendo Educación, Salud y La niña/adolescente. Cabe señalar que únicamente en las investigaciones se destaca especialmente el tema de salud.

La educación de las mujeres hacia la equidad ya no se circunscribe a lo externo sino que se produce una reflexión desde lo interno de las universidades con miras a aumentar la participación de estas en las ciencias. En el ITCR, por ejemplo, tal objetivo concentra alrededor del $90 \%$ de las investigaciones reportadas. 
En la UNED se plantea como proyecto la creación y organización de la Comisión Institucional de Género y, en el ITCR el Plan de Acción: institucionalización de la perspectiva de Género. Más aún, se cuestiona la misma ciencia, tal como se refleja en un proyecto que relaciona la violencia simbólica en la enseñanza del derecho penal (UCR).

Se observan proyectos de información $y$ de recursos sobre temas relacionados con las mujeres, tendientes a la equidad (por ej. Programa de información para la mujer y Centro de Educación especializado de la UNA), los que en ocasiones trascienden el ámbito universitario para constituirse en proyectos de desarrollo (Ej.: Desarrollo integral de las mujeres del Golfo de Nicoya, desde la perspectiva de género, UNA).

En la UCR se plantea la realización de un Perfil Nacional sobre el Desarrollo de las Mujeres, lo que constituye un paso más hacia la auditoría social, investigación-acción y perspectivas de cambio.

El concepto de salud se amplía a temas como la evaluación de servicios, salud reproductiva $y$ VIH-SIDA.

También se presentan trabajos relacionados con el movimiento de mujeres, inclusive dentro de la región centroamericana y más que descripción de situaciones se revela la tendencia al análisis hacia el cambio. El título sugestivo "Auditoría social de derechos humanos de las mujeres como fuerza de trabajo" (UCR) nos indica una nueva pauta participativa.

La recuperación de las voces acalladas se observa claramente en el título "No solo queremos hablar, queremos que nos oigan: derecho a la participación de niñas, niños y adolescentes" (UCR).

La apertura de la Cátedra de Teología Emilia Prieto en la Universidad Nacional fomenta el desarrollo del análisis religioso.

Los estudios sobre masculinidad, aunque incipientes, también se presentan en estos años, reflejando la necesidad de reflexionar sobre esta construcción social.

Mención aparte merece el programa Palabra de Mujer de la UCR, que, si bien se incluye como un solo proyecto en realidad más parecieran varios, pues se trata de un conjunto de acciones que investiga $y$ disemina conocimiento por medios audiovisuales, ya que cada tema conduce a nuevas enseñanzas que de inmediato pasan al conocimiento público. Algunos de los temas realizados son concertación, esterilización, jóvenes, afrodescendientes, violencia, cuerpo, trabajadoras domésticas, campesinas, emprendedoras, violencia, prostitución y arte.

La variedad $y$ versatilidad de los programas constituye un buen ejemplo de las diversas visiones que se presentan en la investigación universitaria, la que también es un importante portón para que en la interrelación academiasociedad se compartan problemas y propongan soluciones.

El panorama señalado indica una tendencia al desarrollo de aspectos teóricos, centrados en la conciencia de la superación de la discriminación de género como base de procesos de cambio social, presentando una serie de proyectos que implican una nueva visión de la investigación, la cual aporta desde una nueva visión centrada en la equidad.

Comparando los resultados con las necesidades en Estudios de la Mujer (ver González, 1997) se observa que los temas más solicitados (desarrollo, economía, trabajo) no se han desarrollado de acuerdo con las expectativas. Muy especialmente el tema de pobreza — si bien se hallan investigaciones puntuales - no se presenta con la profundidad que requiere un problema de trascendencia nacional, por lo que deberá ser impulsado en el futuro.

TESIS

Las tesis son en total 94, las que - de acuerdo con la información oficial recibidase restringen a la Universidad Nacional y a la Universidad de Costa Rica, incluyendo las realizadas conjuntamente por medio de la Maestría Regional en Estudios de la Mujer.

La autoría del total es realizada en un $85 \%$ por mujeres y los temas más destacados son: trabajo/economía, poder y política, violencia y familia. El $80 \%$ del total se ubica en alguno de los rubros de la Plataforma Mundial de Acción.

Los temas de las tesis concuerdan directamente con las necesidades señaladas por las 
organizaciones de mujeres, (economía, violencia $y$ poder) lo que indica un especial interés por parte del estudiantado de enfrentar temas de interés e importancia nacional.

Las tesis relacionadas con economía se centran en hogares pobres e incluyen al sector rural. Cabe señalar que en este grupo es difícil dividir cuáles corresponden al rubro de economía y cuáles a pobreza, ya que en general coinciden. Se presentan también rangos tan variados como una tesis en zona urbana-industrial relacionada con trabajo femenino en la industria manufacturera y otra relacionada con el ascenso a puestos gerenciales.

El poder es develado, incluyendo tesis que se dirigen a la estructura de los partidos tradicionales $y$ a la experiencia de mujeres en puestos electivos.

El análisis de la violencia, especialmente la violencia doméstica, cobra gran relevancia en este período, encontrándose tesis sobre el concepto del cuerpo en mujeres abusadas sexualmente, el amar en la mujer incestada, violencia doméstica y discapacidad, modelo de atención primaria dirigido a la violencia de pareja, análisis psicosocial del ciclo de agresión contra la mujer en el vínculo conyugal, violencia doméstica desde la perspectiva de la mujer agredida $y$ otra desde la perspectiva del hombre privado de libertad (agresor), intervención en crisis individual y grupal sobre la problemática de violencia a mujeres agredidas, la violencia en el noviazgo $y$ el hostigamiento sexual.

Los estudios centrados en personas menores de edad, se dedican fundamentalmente a adolescentes, sobre todo en la relación con su cuerpo: embarazo, enfermedades crónicas y anorexia y percepción corporal de adolescentes relacionadas con prostitución (término posteriormente replanteado como explotación sexual comercial).

En la UNA, con la creación de una maestría especializada, se promovieron tesis sobre temas religiosos, incluyendo la perspectiva de género que promueve una visión más crítica para entender las relaciones dentro de las iglesias.

La Maestría Regional en Estudios de la Mujer recoge una gran variedad de temas citados anteriormente, los que son analizados desde una visión feminista y constituyen un avance importante hacia la equidad $y$ hacia la producción del conocimiento superando sesgos androcéntricos.

La productividad $y$ la variedad de las tesis - así como la intuición estudiantil para escoger temas requeridos por las organizacionesplantean la necesidad de reflexionar acerca de como lograr potenciar este importante aporte. La promoción del trabajo interdisciplinario y la motivación para la integración en programas permitiría fomentar investigaciones de alta calidad y compromiso, en forma tal que al finalizar un número de tesis, el problema haya sido estudiado a profundidad, contándose con las bases para propiciar el cambio requerido.

\section{PUBLICACIONES}

LIBROS

La producción de libros asciende a 51, de los cuales el 70\% corresponde a la Universidad de Costa Rica.Las autorías por parte de mujeres es de un $60 \%$ del total, es decir, disminuyen en un $25 \%$ con respecto a las investigaciones y revistas.

Los temas relacionados con cultura y arte sobresalen indicando una tendencia editorial, pero, en este proceso de cuantificación se presenta una distorsión importante ya que la aparición de nombres femeninos en el título no necesariamente indica un avance en Estudios de la Mujer, y más bien pueden responder a tratamientos estereotipados propios de la literatura de siglos pasados. En este sentido los datos deben tratarse con cuidado y más bien sería recomendable un estudio específico analizando los contenidos y modelos presentados. La preponderancia de estos temas, que ascienden a un $70 \%$ del total, señala la opción por publicar literatura más que resultados de investigación, lo que produce una disminución notable en los rubros indicados en la Plataforma Mundial de Acción, ya que sólo el $24 \%$ se logra ubicar en alguna de las doce áreas, la mayoría en Educación.

Entre las publicaciones destacan dos sobre salud sexual y reproductiva, tema invisibilizado y omitido por años. La apertura a 
visiones indígenas, el cuestionamiento de la maternidad idílica tradicional y la edición de las Obras Completas de Eunice Odio, muestran caminos para recuperar y dar a conocer la vida desde las mujeres.

\section{REVISTAS}

La producción universitaria en artículos de revista provenientes de investigaciones asciende a 144 , con un $47 \%$ correspondiente a la Universidad de Costa Rica. La autoría principal de las mujeres retorna al 85\%, tal como lo presentado en los estudios de investigación.

Los temas más publicados se relacionan con educación, cultura, arte y marco teórico/ feminismo. El 60\% de los anteriores pueden ubicarse en temas de la Plataforma Mundial de Acción siendo los temas principales: educación, poder $y$ violencia.

Una tendencia generalizada, tal vez por estar en instituciones de educación superior, es la preponderancia de la pedagogía y capacitación.

Entre los temas relacionados con salud destacan la atención, servicios y el impacto de las políticas de ajuste estructural, incluyéndose aspectos tradicionales como los relacionados con la maternidad, así como aquellos lamentablemente más modernos como el VIH-SIDA.

La violencia se plantea como un hecho que requiere de acciones urgentes en artículos sobre acoso sexual en el trabajo, violencia masculina contra las mujeres, drogas y su relación con la violencia física y emocional contra las mujeres, violencia doméstica y fútbol, el papel de las mujeres, el Estado y la sociedad en la regulación de la violencia en Costa Rica, y la educación para la no violencia.

Los estudios con personas menores de edad resaltan el período de la adolescencia con temas tales como hostigamiento sexual, derechos humanos, salud, identidad y vocación.

Con respecto a la Economía aparecen propuestas de capacitación para el desarrollo, al igual que programas educativos.

Nuevamente destacan los estudios del ITCR sobre mujeres en la ciencias, cuya participación es necesaria para el progreso social.
El concepto de familia se amplía, cuestionándose las nociones estereotipadas de maternidad y paternidad. La gerontología aporta para resaltar las circunstancias especiales de las adultas mayores, presentándose resultados sobre menopausia, experiencia de la pérdida por muerte del cónyuge, autopercepción de mujeres mayores en el área rural y sentido de la vida en personas mayores de 50 años.

En general se observa una mayor tendencia a profundizar aspectos teóricos desde una perspectiva feminista, así como enfrentar tópicos novedosos como el ecofeminismo, el cuestionamiento a la heterosexualidad normativa y el uso del espacio urbano en la lógica patriarcal.

\section{CONCLUSIONES Y RECOMENDACIONES}

La producción universitaria en el quinquenio de fin de siglo asciende a 381 producciones oficiales en Estudios de la Mujer. Los temas más investigados se relacionan con educación, salud, derechos económicos —incluyendo los laborales - poder/política y violencia. Las publicaciones se centran en cultura $y$ arte, educación, marco teórico, poder y violencia contra las mujeres.

Tales resultados, comparados con las necesidades indicadas por las organizaciones que trabajan con mujeres, que indicaban que los temas de interés eran: trabajo, violencia, poder, salud, familia, educación e identidad, reflejan una respuesta adecuada de la academia a la demanda ciudadana.

Hay una cifra similar entre investigaciones oficiales $y$ tesis, ascendiendo a un total de 186 documentos, de los cuales los elaborados por estudiantes como trabajos finales de graduación son más cercanos a las necesidades indicadas por las organizaciones de mujeres.

El estudiantado tiende a optar en sus proyectos por temas de gran interés práctico que requieren de soluciones imperiosas. Interesa así el tema del empoderamiento, sea este en lo económico, lo público y lo privado, es decir: lo descriptivo y teórico se enmarca en el concepto de bienestar y ejercicio de derechos.

Los proyectos de investigación consignados muestran una tendencia hacia la investigación- 
acción, al incluir opciones como la apertura de programas de género o centros de documentación especializados. De lo anterior se deduce la necesidad de abrir el concepto restringido de investigación a una nueva visión bajo la cual el conocimiento se construye por medio de procesos de cambio.

La creación de instancias (maestrías, centros, etc.) sobre temas específicos redunda en un aumento significativo de la producción académica, lo que sugiere la necesidad de abrir estas oportunidades como forma de encarar temas centrales para el desarrollo social, tales como pobreza, ejercicio de derechos, condiciones laborales, etc.

Las publicaciones muestran una diferencia numérica entre libros y revistas, siendo en total 51 y 144 respectivamente. Es en los libros en los que artificialmente pareciera disminuir la producción de mujeres, cuando en realidad se trata de que los hombres utilizan nombres o referencias a mujeres en los títulos, lo que distorsiona la clasificación, ya que lo anterior no indica necesariamente un interés por la equidad. Tal situación se clarifica suficientemente al constatarse una mayoría de novelas.

Las revistas demuestran avance teórico así como un interés por trascender los tópicos usuales y plantear nuevas alternativas de investigación-acción.

Los temas relacionados con el empoderamiento económico no se han desarrollado en este período al ritmo y necesidad indicado por las organizaciones, baste mencionar que sólo se indica una investigación y cuatro tesis sobre pobreza, primer tema de la Plataforma Mundial de Acción. Otros temas encubiertos, tales como salud sexual y reproductiva, grupos étnicos, discapacidad, masculinidad y medio ambiente, comienzan en forma incipiente a abrirse al escrutinio público.

Con respecto a la autoría entre mujeres y hombres, se observan diferencias solamente por la preponderancia de los varones en temas relacionados con deportes $y$ masculinidad, ambos entendibles, sobre todo porque el análisis de género implica una reflexión profunda desde los intereses y estereotipos educativos específicos.

Entre las fortalezas de la producción se aprecia el aumento de estudios con perspectiva de género, el interés por la equidad y el avance hacia los derechos fundamentales, la relación entre necesidades sociales y academia, el desarrollo teórico $y$ de instancias específicas para el cambio a lo interno de las universidades y la conciencia crítica hacia los encubrimientos e invisibilizaciones de problemas.

Como debilidades se encuentra la escasez de trabajos interdisciplinarios, la persistencia de segmentación de la producción —en lugar de una opción diversa e integrada- $y$ sobre todo la carencia de planes de construcción del conocimiento por etapas, partiendo de las grandes problemáticas nacionales.

A pesar de lo anterior persisten vacíos importantes que deben ser enfrentados adecuadamente, entre los más obvios se destacan los relacionados con derechos sexuales y reproductivos, la identidad de género, condiciones de pobreza y su impacto sobre las mujeres y cómo superarla y el desglose de la diversidad, incluyendo la voz de los grupos altamente discriminados tales como afrocostarricenses, indígenas, lesbianas, con discapacidad, migrantes, trabajadoras del sexo, trabajadoras domésticas, adultas mayores y jóvenes.

Considerando los retos citados se presenta una fuerte demanda para impulsar actividades para:

$\diamond$ Continuar relaciones que produzcan sinergia entre academia, movimiento de mujeres y Estado, hacia la equidad de género.

$\diamond$ Desarrollar teóricamente un nuevo paradigma del conocimiento, basado en los derechos e inclusivo de la diversidad.

$\diamond$ Concientizar sobre la importancia de la participación ciudadana, la transparencia y la rendición de cuentas en la democracia.

$\diamond$ Evaluar la calidad de los servicios y del impacto de los programas de gobierno desde la perspectiva de género.

$\diamond$ Desarrollar estadísticas con perspectiva de género y de diversidad.

$\diamond$ Dar seguimiento sistemático al cambio social -especialmente el impacto del neoliberalismo y la globalización económicasobre la vida de las mujeres.

$\diamond$ Evaluar la pérdida de derechos señalando alarmas y medidas correctivas. 
$\diamond$ Investigar, valorar, difundir y supervisar el cumplimiento de los derechos de las mujeres, sobre todo de grupos vulnerables.

$\diamond$ Evaluar y penalizar el incumplimiento de los derechos laborales y proponer la ampliación de los mismos para la igualdad de oportunidades. Proponer y evaluar políticas de empleo y desarrollo económico.

$\diamond$ Verificar el cumplimiento de los derechos económicos y ambientales/ecológicos (crédito, tenencia de la tierra, desarrollo sostenible, etc.) con equidad.

$\diamond$ Fomentar la paternidad responsable y el cambio en las relaciones de explotación doméstica, incluyendo aspectos económicos (pensiones alimenticias), como emocionales.

$\diamond$ Visibilizar las demandas de grupos altamente discriminados tales como afrocostarricenses, campesinas, indígenas, lesbianas, con discapacidad, migrantes, trabajadoras del sexo, trabajadoras domésticas, adultas mayores y jóvenes.

$\diamond$ Continuar impulsando programas contra la violencia hacia las mujeres.

$\diamond$ Desarrollar programas de educación sexual, desde una perspectiva de derechos.

$\diamond$ Proponer servicios de salud de calidad, con perspectiva de género, incluyendo salud reproductiva y muy especialmente el impacto de la ilegalidad del aborto en la salud pública.

$\diamond$ Desarrollar investigación sobre indicadores de salud de las mujeres, sobre el costo personal y social del cuido familiar y labores domésticas, valorando la calidad del servicio y la humanización de los mismos. Desarrollar investigación sobre cáncer, menopausia, parto y posparto y VIH-SIDA.

$\diamond$ Proponer programas para un cambio a corto plazo de patrones de explotación y violencia en las relaciones de género.

$\diamond$ Rescatar, visibilizar y potenciar las iniciativas de las mujeres relacionadas con el cuido ecológico y el desarrollo sostenible.

Cabe señalar que prácticamente no existen propuestas para el disfrute de la vida de las mujeres, tiempo libre y recreación, lo que puede interpretarse como la priorización por las necesidades más urgentes olvidando que la alegría y la expansión también requieren sus espacios y son parte de la salud integral.

Finalmente, un estado democrático requiere de participación popular, transparencia, evaluación permanente y rendición de cuentas, lo que a su vez implica la producción de datos confiables que permita verificar el avance o retroceso según las metas planteadas. De acuerdo con lo anterior la participación de diversas instancias -Estado, organizaciones sociales y academia- es imprescindible para potenciar el cambio hacia el desarrollo en el marco del ejercicio de los derechos humanos.

\section{REFERENCIAS}

Agenda Política de Mujeres. Agenda Política de Mujeres Costarricenses. San José: Grupo Agenda Política de Mujeres, 1997.

Aguilar, Ana Leticia et al. Movimiento de mujeres en Centroamérica. Managua: Programa Regional La Corriente, 1997.

Arroyo, Roxana; Grau, Ariane; Ugalde, Yamileth; Madden, Rose Mary; Quesada, Lili y Solano, Marta. Informe alternativo sobre el cumplimiento de la Plataforma de Acción Mundial de la IV Conferencia Mundial de las mujeres por parte del gobierno costarricense. Iniciativa Centroamericana de Seguimiento a Beijing. Capítulo de Costa Rica, San José, 1999.

Berrón, Linda (Comp.). Las mujeres y el poder. San José: Editorial Mujeres, 1997.

Campbell, Epsy; Escalante, Ana C. y Seas, Flor. Construyendo una Centroamérica con equidad. Foro de Mujeres para la Integración Centroamericana San José, 1997.

Centro de Estudios y Publicaciones Alforja. Iniciativa Centroamericana de seguimiento a Beijing. La Plataforma de acción para las mujeres de Beijing a 
5 años. Una mirada desde las mujeres de los sectores sociales. San José.CEPALFORJA, 2000.

Centro Nacional para el Desarrollo de la Mujer y la Familia. De la Plataforma de Acción a la Plataforma Nacional a través de planes nacionales. Los compromisos de Beijing y su cumplimiento en Costa Rica. San José: CMF, 1997.

Defensoría de los Habitantes. Compendio de Resoluciones de la Defensoría de la Mujer. San José: Defensoría de la Mujer, 2000.

González Suárez, Mirta. Estudios de la Mujer: conocimiento y cambio. San José: EDUCA 1988.

"Necesidades de la investigación en Estudios de la Mujer en Centroamérica". En: Actualidades de Psicología. Vol. 13, número 95, Universidad de Costa Rica: Instituto de Investigaciones Psicológicas, 1997.

Guzmán Stein, Laura. "El estado de la investigación en Estudios de la Mujer en la Universidad de Costa Rica". Informe de investigación, Universidad de Costa Rica, mimeo, 1999.
InAmu. Balance del Estado de Costa Rica de la implementación de la Plataforma de Acción de la Cuarta Conferencia Mundial sobre la Mujer. San José: INAMU, 1999.

Iniciativa Centroamericana de Seguimiento a Beijing. Informe alternativo sobre el cumplimiento de la Plataforma de Acción mundial de la IV Conferencia Mundial de las mujeres por parte del gobierno costarricense. San José, 1999.

Iniciativa Raíces de la Diversidad. La Plataforma de Acción de la IV Conferencia Mundial de la Mujer: una mirada desde la diversidad en Costa Rica. San José: FIRE, UNIFEM, HIVOS, 2000.

Leitenger, Ilse Abshagen. The Costa Rican Women's Movement. Pittsburgh: University of Pittsburgh Press, 1997.

Maestría Regional en Estudios de la Mujer UNA/ UCR. Memorias Taller "Mujer, participación política y ciudadanía”. San José, 1997.

Naciones Unidas Plataforma de Acción. Declaración de Beijing. Información General y selección de Documentos. San José: CMF y Ministerio de Planificación y Política Económica, 1996. 